\title{
Impact of antenatal glucocorticoid exposure on the activity of the stress system, cognition and behavior in 8 to 9-year-old children: a clinical cohort study
}

\author{
Florian Rakers ${ }^{1}$, Ekkehard Schleussner ${ }^{1}$, Isabel Muth ${ }^{1}$, Dirk Hoyer ${ }^{1}$, Sven Rupprecht ${ }^{1}$, \\ Karin Schiecke ${ }^{1}$, Tanja Groten ${ }^{1}$, Michelle Dreiling ${ }^{1}$, Valeska Kozik ${ }^{1}$, Matthias Schwab ${ }^{1}$, \\ Heike Hoyer $^{1}$, and Carolin Ligges ${ }^{1}$ \\ ${ }^{1}$ Jena University Hospital
}

December 13, 2021

\begin{abstract}
Objective: To determine stress-sensitivity and neurodevelopmental outcome in 8- to 9-year-old children following antenatal exposure to glucocorticoid (GC) prophylaxis for neonatal respiratory distress syndrome. Design: Clinical cohort study. Setting: University-based obstetric clinic in Central Germany. Population: 31 term or near-term born children whose mothers received single or multiple courses of betamethasone (BM) to induce fetal lung maturation in threatened preterm birth compared to 39 non-exposed children. Methods: Multi-system assessment of the individual stress response together with an analysis of cognitive, behavioral and electrocortical functioning. Main Outcome Measures: Activity of the hypothalamus-pituitary-adrenal axis (HPAA, primary outcome domain) and the autonomic nervous system (ANS, secondary outcome domain) including markers of heart rate variability (HRV). Additional endpoints were the cognitive performance (IQ) and attention-deficit/hyperactivity disorder (ADHD) core symptoms. Results: HPAA activity was not affected by antenatal GC-exposure. ANS activity in GCexposed children shifted towards a higher parasympathetic tone reflected by a higher overall high-frequency band power of HRV (1313 vs. $762 \mathrm{msec} 2 / \mathrm{Hz}, \mathrm{p}=0.03$ ). BM-exposed children had lower cognitive performance (IQ 96.9 vs. 108.0, p<0.01) and a marginally higher ADHD score (FBB-ADHD scale 5.5 vs. 4.6 points, p=0.04). A monotonic dose-response relationship between GC-exposure and stress-induced activity of the ANS and IQ was estimated post-hoc. Conclusions: Antenatal exposure to supraphysiological concentrations of BM in the context of threatened preterm birth was associated with multidimensional changes in stress-sensitivity and neurodevelopment in later life. As these changes may be dose-dependent, antenatal GC prophylaxis should be used at the minimum effective dose after a careful risk-benefit assessment.
\end{abstract}

Impact of antenatal glucocorticoid exposure on the activity of the stress system, cognition and behavior in 8 to 9 -year-old children: a clinical cohort study

Florian Rakers ${ }^{1}$, Ekkehard Schleußner ${ }^{2}$, Isabel Muth ${ }^{2}$, Dirk Hoyer ${ }^{1}$, Sven Rupprecht ${ }^{1}$, Karin Schiecke ${ }^{3}$, Tanja Groten $^{2}$, Michelle Dreiling ${ }^{1}$, Valeska Kozik ${ }^{1}$, Matthias Schwab ${ }^{1}$, Heike Hoyer ${ }^{3 \#, ~ C a r o l i n ~ L i g g e s ~}{ }^{4 \#}$

\# authors contributed equally to the study

${ }^{1}$ Hans Berger Department of Neurology; ${ }^{2}$ Department of Obstetrics $;{ }^{3}$ Institute of Medical Statistics, Computer and Data Sciences, ${ }^{4}$ Department of Child and Adolescent Psychiatry, Psychosomatic Medicine and Psychotherapy; Jena University Hospital, Jena, Germany

\section{Corresponding author:}

PD Dr. Florian Rakers, Hans Berger Department of Neurology, Jena University Hospital, Am Klinikum 1, 07747 Jena, Germany, Email: florian.rakers@med.uni-jena.de, Phone: +49-3641-9323467 
Short title: Antenatal Glucocorticoids and Neurodevelopment

\begin{abstract}
Objective: To determine stress-sensitivity and neurodevelopmental outcome in 8- to 9-year-old children following antenatal exposure to glucocorticoid (GC) prophylaxis for neonatal respiratory distress syndrome.

Design: Clinical cohort study.

Setting: University-based obstetric clinic in Central Germany.

Population: 31 term or near-term born children whose mothers received single or multiple courses of betamethasone (BM) to induce fetal lung maturation in threatened preterm birth compared to 39 nonexposed children.
\end{abstract}

Methods: Multi-system assessment of the individual stress response together with an analysis of cognitive, behavioral and electrocortical functioning.

Main Outcome Measures: Activity of the hypothalamus-pituitary-adrenal axis (HPAA, primary outcome domain) and the autonomic nervous system (ANS, secondary outcome domain) including markers of heart rate variability (HRV). Additional endpoints were the cognitive performance (IQ) and attentiondeficit/hyperactivity disorder (ADHD) core symptoms.

Results: HPAA activity was not affected by antenatal GC-exposure. ANS activity in GC-exposed children shifted towards a higher parasympathetic tone reflected by a higher overall high-frequency band power of HRV (1313 vs. $762 \mathrm{msec}^{2} / \mathrm{Hz}, \mathrm{p}=0.03$ ). BM-exposed children had lower cognitive performance (IQ 96.9vs. 108.0, $\mathrm{p}<0.01$ ) and a marginally higher ADHD score (FBB-ADHD scale 5.5 vs. 4.6 points, $\mathrm{p}=0.04$ ). A monotonic dose-response relationship between GC-exposure and stress-induced activity of the ANS and IQ was estimated post-hoc.

Conclusions: Antenatal exposure to supraphysiological concentrations of BM in the context of threatened preterm birth was associated with multidimensional changes in stress-sensitivity and neurodevelopment in later life. As these changes may be dose-dependent, antenatal GC prophylaxis should be used at the minimum effective dose after a careful risk-benefit assessment.

\title{
Introduction
}

The high plasticity of the fetal central nervous system (CNS) characterises a significant success factor of human evolution; however, it also represents one of its greatest challenges. It is now becoming increasingly evident that unfavorable intrauterine conditions can alter the developmental trajectories of organs with high plasticity, above all the fetal CNS. This observation has previously been termed 'the fetal programming of health and disease' 1 .

One of the best characterized mediators of fetal CNS programming are glucocorticoids (GCs) resulting either from maternal psychosocial stress or - amongst others - from GC prophylaxis with synthetic GCs to induce fetal lung maturation in women threatened by premature birth. Supraphysiological concentrations of GCs are known to interfere with many aspects of fetal structural and functional brain development ${ }^{2-4}$ including the maturation of the fetal stress system with its two branches: the hypothalamus-pituitary-adrenal axis (HPAA) with its main effector hormone cortisol ${ }^{5}$ and the autonomic nervous system ${ }^{6}$ (ANS). Dysregulation of an individuals' stress system is thought to underlie a number of cognitive and behavioral disorders in later life, including cognitive dysfunction and attention-deficit/hyperactivity disorder (ADHD) ${ }^{6-8}$.

While GC-induced fetal programming of the stress system and its cognitive and behavioral consequences in later life have been well characterized in several animal species, including rodents, sheep, and non-human primates ${ }^{5}$, systematic research on human subjects is scarce and primarily limited to the programming of the activity of the HPAA ${ }^{9,10}$. It is still unclear if fetal exposure to high levels of GCs in humans also programs long-lasting dysregulation of the ANS and increases the risk for cognitive and behavioral deviations in later life. 
In this study, we examined the programming effects of antenatal GCs in 8-to 9-year-old offspring exposed to antenatal GC prophylaxis to induce fetal lung maturation in the context of threatened preterm birth compared to a non-exposed control group. We conducted a multi-system assessment of the individual stress response together with an analysis of cognitive, behavioral and electrocortical functioning. Our primary hypothesis was that antenatal exposure to BM would exacerbate the HPAA response to acute stress in later life as seen in animal experiments ${ }^{5}$. We assumed that antenatal BM treatment would also affect the autonomic stress response. In addition, we expected changes in cognitive performance, presence of ADHD-like behavior, and altered electrocortical brain function.

\section{Materials and methods}

\section{Recruitment strategy and study subjects}

The recruitment process is visualized in Figure S1.

\section{BM-group}

We invited 105 mother-child pairs to participate in the current study. Whilst pregnant, these mothers had taken part in a randomized controlled interventional trial that compared the effects of two tocolytic treatments for threatened premature birth ${ }^{11}$. During this trial, as part of routine clinical management, children were antenatally exposed to single or multiple courses of $2 \times 8 \mathrm{mg}$ doses of BM $24 \mathrm{~h}$ apart to induce fetal lung maturation but born near or on-term.

Exclusion criteria for children in the BM-group included a birth weight of less than the $5^{\text {th }}$ reference percentile $^{12}$, severe perinatal complications, continuous GC treatment, exposure to maternal smoking, alcohol or drugs in utero as well as incomplete records of birth data. Of the 105 mother-child pairs invited, 42 pairs agreed to participate. Three children met one or more of the exclusion criteria (see Figure S1 for details) and were excluded from the study. The remaining 39 children were enrolled in our study and formed the BM-group.

\section{Control group}

Initially, we matched children in the control group by current age (+/-3 months), gender, and gestational age at birth (+/-14 days) at a ratio of 1:1. Exclusion criteria were the same as for the BM-group. The children for the control group were identified from hospital medical records of births. A total of 53 mother-child pairs agreed to participate in our study. Eight children met the exclusion criteria of which five had incomplete birth records. Six children had no matching partner in the BM-group. Thus, 39 children were enrolled in the control group.

Due to additional information received only after outcome assessment, eight children from the BM-group and one child from the control group had to be excluded from the analysis (Figure S1). The final analysis was comprised of data from 31 children in the BM-group and 38 children in the control group.

\section{Demographic and clinical baseline data}

The family's socioeconomic status (SES) together with other demographic characteristics was collected using self-developed questionnaires. Pregnancy and birth data were determined using information documented in the Maternal and Child Handbook.

\section{Outcomes of interest}

The primary outcome domain was the neuroendocrine stress response specified as the activity of the HPAA expressed as salivary free cortisol at rest and during acute stress. The secondary outcome domain was the activity of the ANS during acute stress and comprised markers of heart rate variability (HRV), baroreflex sensitivity and salivary $\alpha$-amylase concentration. Other endpoints were the cognitive performance expressed as the intelligence quotient (IQ), presence of ADHD core symptoms and electrocortical activity measured via EEG signals. 


\section{Study procedure and outcome assessment}

Assessment of the function of the stress system

To evaluate the response of the stress system to acute stress, children were exposed to the Trier Social Stress Test version adapted for children (TSST-C) ${ }^{13}$. The TSST-C is a validated, standardized stress protocol that reliably provokes activation of the HPAA ${ }^{9}, 10,14,15$ and $\mathrm{ANS}^{16}$ in children and includes a preparation / baseline period, a speech and mental arithmetic task in front of an audience and a recovery period. During the TSST-C, the child was hooked up to a polysomnography device (SOMNOscreen plus, SOMNOmedics GmbH, Germany) to obtain continuous EEG, ECG and systolic blood pressure recordings. The course of the TSST-C is shown in Figure 1 and follows the original protocol ${ }^{13}$ with the difference that the children in the present study were additionally attached to a stationary EEG, to ECG electrodes and to a blood pressure finger-cuff. This means that, in contrast to the original protocol ${ }^{13}$, the children were already in the examination room during the preparation period of the TSST-C. Four saliva samples (Salivettes, Sarstedt, Nümbrecht, Germany) were collected before, during, and after the TSST-C (see Figure 1 for timepoints) to determine salivary free cortisol and $\alpha$-amylase as surrogate markers of HPAA ${ }^{17}$ and sympathetic ANS $^{18}$ activity, respectively. To estimate HPAA activity at rest, parents were asked to collect two saliva samples of their child at home during a restful day.

\section{Hormone analysis}

The concentration of salivary cortisol was estimated using a commercially available chemiluminescence immunoassay (RE62011, IBL International GmbH, Hamburg, Germany) in accordance with the manufacturer's protocol with an intra- and interassay coefficient of variation (CV) of $4.5 \%$ and $4.3 \%$, respectively. The concentration of the saliva $\alpha$-amylase was estimated by means of an enzymatic colorimetric assay using an autoanalyzer (ARCHITECT c4000; kits 30-3951/R6; ABBOTT laboratories, Wiesbaden, Germany) with an intra- and interassay CV of $1.2 \%$ and $2.8 \%$, respectively.

Analysis of heart rate variability (HRV) and baroreflex sensitivity (BRS)

ANS activity was estimated by analyzing the autonomic control of the cardiovascular function using HRV frequency indices (low frequency (LF) and high frequency (HF) band power, LF/HF ratio) calculated from the continuous ECG-recording for each part of the TSST-C ${ }^{19}{ }^{20}$. BRS was assessed from LF and HF power spectra of pulse intervals and systolic blood pressure (alpha index) obtained from the continuous blood pressure recordings ${ }^{21}$.

\section{Psychological assessment}

Cognitive performance was assessed using the Raven's Colored Progressive Matrices test (CPM, nonverbal assessment of general intelligence $)^{22}$. To assess the core ADHD-symptoms of inattention, hyperactivity and impulsivity, we used the parental questionnaire for the assessment of ADHD (FBB-ADHS) via 20 items according to ICD-10 and DSM-IV diagnostic criteria ${ }^{23}$.

\section{Analysis of the EEG}

We determined the spectral edge frequency (SEF) of the total band power $(1.5-30 \mathrm{~Hz})$ of the continuous EEG recordings for each part of the TSST-C. The SEF is a sensitive measure of cortical activity and a physiological correlate of global brain function ${ }^{24,25}$. It is defined as the frequency below which $95 \%$ of the power resides ${ }^{26}$. The software used for the analysis comprised WATISA (Institute of Medical Statistics, Computer and Data Sciences, University of Jena, Germany).

\section{Statistical analysis}

Based on a small pilot study (data on file), a sample size of 40 children per group was planned. This sample size would have $80 \%$ power to detect a standardized effect size (SE) of [?]0.63 using a two-group t-test with a two-sided significance level of 0.05 . The characteristics of the participants and the socioeconomic data are displayed in exposure groups by adequate statistical measures. We included the graduate degree 
of at least one parent (no/yes) as proxy covariate in all analyses to account for differences in the SES. To reflect the design, mixed linear models were used to account for matching and, if appropriate, for repeated measurements during TSST-C. Exposure to BM, the graduate degree of parent(s) and, if appropriate, the TSST-C period were fixed factors. Two different models were fitted to analyze the main effect of exposure to BM on the TSST-C stress response. The overall-activity model comprised outcomes of all TSST-C periods including baseline and feedback. We used the change-from-baseline model to analyze the baseline adjusted differences between active periods of TSST-C and baseline. Natural log transformation was used for nonnormal data. Estimated outcomes are presented as arithmetic means and their differences or geometric means and their ratios (for back-transformed log-data) with $95 \%$ confidence intervals (CI), respectively. Moreover, effects were standardized in a similar way to Cohens' $d{ }^{27}$. Statistical testing and presentation of p-values was restricted to the comparison of outcomes. The two-sided significance level was 0.05. The dose-effect relationship was explored post hoc by contrasting children in the BM-group exposed to multiple and single courses of BM with controls. For this purpose, effect measures with multiplicity adjusted $95 \%$ CIs (Dunnett-Hsu) are presented. All analyses were performed using SAS 9.4.

\section{Results}

Demographic and clinical baseline data

Both study groups were similar for age gender and gestational age at birth (Table 1). All other non-matched variables were also well-balanced between the groups except for the SES (Table 1) which we used as a single proxy variable in the outcome analysis to adjust for this imbalance.

Exposure characteristics

12 out of 31 children were exposed to repeated courses of BM. Exposure characteristics are described in detail in Table S1.

\section{Outcomes}

Primary outcome domain

Neuroendocrine stress response

Salivary cortisol concentration did not differ significantly between BM-group and controls at rest and in response to acute stress (Figure 2, Table 2). The standardized effect (SE) for non-significant effects ranged from 0.1 to 0.49. The highest of these SEs was found for the afternoon resting cortisol concentration in BMgroup (2.35 vs.1.75 nmol/l, $\mathrm{p}=0.07, \mathrm{SE}=0.49$, Table 2). A similar $\mathrm{SE}$ was found for the cortisol fold-change from baseline during the TSST-C which tended to be lower in BM-group (1.33 vs. $1.76, \mathrm{p}=0.13, \mathrm{SE}=0.39$, Table 2).

Secondary outcomes

ANS stress response:

Saliva $\alpha$-amylase concentration did not differ significantly between BM-group and controls (Table 2).

Overall ANS activity: The HF-band power during the TSST-C (all timepoints T1 - T5) was higher in BMgroup compared to controls (1313vs. $762 \mathrm{msec}^{2} / \mathrm{Hz}, \mathrm{p}=0.03, \mathrm{SE}=0.57$, Table 2) resulting in a lower LF/HF ratio in BM-exposed children (LF/HF ratio during the TSST-C 1.30 vs. 1.86, $\mathrm{p}<0.01, \mathrm{SE}=0.75$, Table 2, Figure S2).

Stress-induced ANS activity: Stress-induced changes from baseline (timepoints T1 vs. T2-T4) in HF band power $\left(-451\right.$ vs. $-1192 \mathrm{msec}^{2} / \mathrm{Hz}, \mathrm{p}=0.03, \mathrm{SE}=0.56$, Table 2$)$ and $\mathrm{LF} / \mathrm{HF}$ ratio $(0.33$ vs. $1.05, \mathrm{p}<0.01$, $\mathrm{SE}=1.05$, Table 2, Figure S2) were less marked in BM-group vs. controls. The SE for non-significant effects ranged from 0.08 to 0.49 . The stress induced increase in heart-rate tended to be lower in the BM-group (5.08vs. 8.85 bpm, $\mathrm{p}=0.06, \mathrm{SE}=0.49$, Table 2).

Cognition and behavior 
BM-exposed children had significantly lower scores for cognitive performance (IQ 96.9 vs. 108.0, $\mathrm{p}<0.01$, $\mathrm{SE}=0.68$, Table 2, Figure S3) and higher ADHD-scores (stanine 5.5 vs . 4.6, $\mathrm{p}=0.04, \mathrm{SE}=0.51$, Table 2, Figure S3). However, all 95\%-CIs were still within the normal range expected for the age examined.

\section{Electrocortical function}

Overall electrocortical activity: The overall SEF at the occipital electrodes during the TSST-C (all timepoints $\mathrm{T} 1$ - T5) was higher in the BM-group than in controls $(15.5$ vs. $14.1 \mathrm{~Hz}, \mathrm{p}=0.02$, $\mathrm{SE}=0.56$, Table 2, Figure $\mathrm{S} 4)$. A slightly smaller effect was observed for the temporal electrodes $(18.7$ vs. $17.2 \mathrm{~Hz}, \mathrm{p}=0.07, \mathrm{SE}=0.44$, Table 2, Figure S4). The higher SEF can be explained by a higher relative band power of beta waves (data not shown). No significant differences in the SEF were detected between both groups at the frontal and parietal electrodes.

Stress-induced electrocortical activity: There were no significant differences in the SEF-change from baseline between both groups. The standardized size of the non-significant effects ranged from 0.08 to 0.39 .

Effect of multiple BM-courses (post-hoc analysis)

In an exploratory post-hoc analysis, a monotonic dose-response relationship was observed between BMexposure and the stress-induced activity of the ANS and cognitive performance (IQ). LF/HF ratio change from baseline decreased from 1.05 for controls to 0.40 and 0.19 for single and multiple courses of BM, respectively. The IQ decreased from 108.0 for controls to 101.7 for single courses and to 89.3 for multiple courses of BM. Furthermore, a trend was observed towards a lower cortisol fold change from baseline during the TSST-C (controls: 1.76, single course: 1.51, multiple courses: 1.05) and towards a higher overall activity of the SEF at the temporal $(17.2,18.2,19.6 \mathrm{~Hz})$ and occipital $(14.1,15.3,15.8 \mathrm{~Hz})$ electrodes. For details and group differences, see Table $\mathrm{S} 2$.

\section{Discussion}

The present study investigates the effects of antenatal GC exposure on the neuroendocrine and autonomic stress system and on markers of cognitive and behavioral functioning. In contrast to our primary hypothesis, antenatal BM-exposure was not associated with significant changes with regard to neuroendocrine activity of the stress system at the age of 8-9 years. However, our results indicate a programming effect of BM on the autonomic stress system as well as on brain development and function in later life.

Neuroendocrine stress response - HPAA activity

In contrast to our primary hypothesis, HPAA activity during the TSST-C did not differ significantly between BM-group and controls or tended even to be lower in BM-exposed children. To date, only one longitudinal study has investigated the impact of antenatal BM exposure on offspring HPAA activation in term-born individuals ${ }^{9,10}$. In contrast to our study, Alexander et. al found a more pronounced increase in cortisol concentration in response to the TSST-C in BM-exposed vs. non-exposed children aged 6 to $11^{9}$ and in adolescents aged 14 to $18^{10}$. A potential explanation for these differences might be found in the varying dosing regimens of $\mathrm{BM}$ used in the different studies. While Alexander et al. only included children antenatally exposed to a single course of BM, our study also included children exposed to repeated courses of BM reflecting real-life prescription patterns ${ }^{28}$. Repeated, but not single courses of BM, are known to cause HPAA hyporesponsiveness in healthy human neonates ${ }^{29}$ and in adult sheep ${ }^{30}$; the latter represents the standard animal model of human pregnancy. Similarly, antenatal treatment with the high-potency GC dexamethasone, but not the low-potency GC hydrocortisone, resulted in blunted HPAA activation during the TSST-C ${ }^{31}$ in prematurely born children. To further explore a potential dosing effect of BM on HPAA reactivity, we performed an exploratory post-hoc analysis. Indeed, a trend towards a negative dose-effect relationship between BM exposure and HPAA reactivity was estimated, supporting the assumption that the change in the direction of the BM-induced activity of the HPAA depends on the cumulative dose of antenatal BM-exposure. Differences in HPAA reactivity may also be attributed to our adaptation of the original TSST$\mathrm{C}$ protocol that resulted in a moderately lower overall HPAA activation compared to previous studies ${ }^{9,10}$ and may have masked a potential HPAA hyperresponsiveness. Nevertheless, as the HPAA reactivity in the 
BM-group tended to be even lower compared to controls, a more pronounced stress exposure would most likely not have inverted our results.

Consistent with the results of other human cohort studies of subjects exposed to prenatal GC prophylaxis, basal HPAA activity determined at different timepoints on a restful day did not differ between the BM group and controls ${ }^{9}, 10,32-34$ suggesting that BM at the dose and timing used in our study does not modify the basal set point of the HPAA.

\section{Secondary outcome measures}

\section{ANS stress response}

HF band power during the TSST-C was higher in the BM group and its stress-induced decrease was less pronounced than in controls. As the efferent cardiac vagal nerve activity is the major contributor to the HF band power ${ }^{35-37}$, this finding suggests an overall increased vagal tone in BM-exposed children. Moreover, because during normal infant development, parasympathetic activity gradually increases ${ }^{6}$, a higher vagal tone in BM-exposed children likely reflects BM-induced accelerated maturation of the parasympathetic ANS. Furthermore, because the power of the LF band did not differ between the groups, whereas the power of the $\mathrm{HF}$ band was increased in the BM group, the LF/HF ratio was lower in the BM group than in controls. The $\mathrm{LF} / \mathrm{HF}$ ratio is thought to represent the sympatho-vagal balance as it is assumed that sympathetic nerve activity is the main factor modulating the LF component of HRV ${ }^{35}$. In this context, our results suggest a parasympathetic shift of the sympatho-vagal balance in the BM-group and an exclusive effect of BM on the development or function of the parasympathetic ANS. In agreement with this observation, saliva $\alpha$-amylase as a humoral marker of the sympathetic activity ${ }^{18}$ did not differ between groups. However, it has been suggested that the sympatho-vagal balance cannot be accurately measured via the LF/HF ratio ${ }^{37}$, hence interpretation of our data merits some caution.

\section{Cognition and behavior}

IQ as a marker of general cognitive function was within the expected normal range in both groups. Within this normal range, IQ in the BM-group was on average 11.1 points lower compared to controls. In contrast to our results, previous follow-up studies of several well-controlled RCTs did not reveal any impact of antenatal GC-prophylaxis on cognitive performance in individuals aged $4-31$ years of age ${ }^{38-42}$. Differences may be attributed to the cumulative dose of BM administered. All studies cited above included children exposed to one course of BM whereas we also included children exposed to multiple courses. Indeed, in our exploratory post-hoc analysis, a negative dose-effect relationship was observed with lowest IQ scores seen in children exposed to multiple BM courses.

Parent-reported ADHD-scores were within the expected normal range in both groups. Within this normal range, BM-exposed children displayed a higher ADHD-score than controls. A higher risk of mental and behavioral disorders including ADHD was also reported in a large population-based cohort study from Finland following antenatal exposure to maternal GC prophylaxis ${ }^{43}$. It should be noted, however, that not all available studies found a significant effect of antenatal BM-exposure with respect to ADHD symptoms 38,39 .

\section{Electrocortical activity}

Compared to controls, children in the BM-group displayed a higher overall electrocortical activity determined by power spectral analysis of EEG signals and calculation of the SEF which was accentuated in the occipital brain regions. The SEF represents an effective estimate of the frequency content of the EEG power spectrum 44. During physiological development, the proportion of higher frequencies in the EEG power spectrum increases from childhood to adolescence, reflecting maturational changes of thalamo-cortical and corticocortical networks ${ }^{45}, 46$. The higher frequencies start to develop in the occipital brain regions first ${ }^{45}$. Thus, a higher SEF at the occipital electrodes observed in BM-exposed children is suggestive of GC-induced maturational acceleration of subcortical and cortical structures ${ }^{45,46}$. In agreement with our study, in preterm infants, antenatal GC prophylaxis was negatively associated with EEG slow delta and theta activity 
at rest ${ }^{47}$. Animal studies in fetal sheep revealed lasting complex changes in neuronal activity after GC administration ${ }^{48}$ with some reports of increased high frequencies ${ }^{49}$.

\section{Strength and limitations}

Our study included only healthy children born at or near-term who were reasonably matched with regard to gestational age at birth. Thus, in contrast to previous studies examining the individual stress responsiveness in terms of HPAA ${ }^{15,29,31-34}$ or ANS activity ${ }^{32,50}$ and cognition and behavior ${ }^{38-42}$ following antenatal GCexposure, we can exclude an effect of prematurity on our outcome measures ${ }^{51}$. In addition, we considered parental SES as a potential confounder that has not been considered in most studies to date. Parental SES is independently associated with stress reactivity, cognition and behavior in later life ${ }^{52-54}$. As we noticed a significant difference in SES between BM-group and controls potentially reflecting participation bias, we statistically adjusted for this imbalance. Like other studies before, we cannot separate the direct effects of GCs from those caused by prenatal maternal stress associated with the risk of preterm birth ${ }^{1}$. Future studies may add a separate control group with comparable maternal stress elicited for example by adverse life events to overcome this issue. We cannot rule out an independent effect of the tocolytic treatment on our outcome measures. Because two pharmacologically different tocolytics were used in a balanced manner, a significant bias is unlikely. Due to post-matching exclusions, sample size and precision of the effect estimates were lower than planned. We did not adjust the level of significance for multiple outcomes in our exploratory study which raises the risk of false-positive results. Our post hoc analysis on dosing effects of BM is only exploratory. Observed effects may also be attributed, in part, to maternal stress associated with a prolonged hospital stay necessary to receive multiple BM courses. The observational study design does not allow to conclude a causal relationship between BM exposure and our end points. In addition, it is not possible to assess the extent to which the postnatal environment influenced the outcome variables. Given the complex and long-lasting nature of brain development, we acknowledge that some residual confounding is likely.

\section{Conclusion}

Considering the limitations described above, our results suggest that antenatal exposure to supraphysiological concentrations of BM for threatened premature birth seems to be associated with long-lasting, multidimensional changes in stress sensitivity and neurodevelopment 8 to 9 -year-old children. These alterations are expressed as a reduction in cognitive performance and a slight increase in ADHD-scores. Cognitive and behavioral changes are accompanied by electrophysiological correlates of aberrations in cortical and subcortical brain function.

We found hints for a monotonic dose-effect relationship for all outcome domains except behavior suggesting that GC-induced programming of fetal neuronal functioning may be dose-dependent. We propose that, in spite of the irrefutable benefit of GC prophylaxis for the short-term outcome in very preterm infants, antenatal GCs in obstetrics and other clinical indications should only be prescribed at the minimum effective dosage after a careful risk-benefit assessment.

\section{Acknowledgements}

We thank Jana Ziegler and Torsten Schulze for their technical support and Nasim Kroegel for her critical reading and language editing of the manuscript.

\section{Disclosure of Interests}

The authors have nothing do disclose.

\section{Contribution to Authorship}

The idea of this study was conceived by MS and ES. IM, SR, and CL recruited the participants and performed the tests. DH, KS and HH analyzed the data. FR interpreted the data and wrote the first draft of the manuscript. VK, MD and TG contributed to the interpretation of the data and wrote specific parts of the manuscript. All authors critically revised the manuscript. 


\section{Details of Ethics Approval}

The study protocol and all procedures were approved by the ethics committee of Jena University Hospital for the study period October 2008 to August 2009 (Approval 2041-06/07).

\section{Funding}

This study was funded by the intramural research grant of Jena University Hospital.

\section{References}

1. Van den Bergh BRH, van den Heuvel MI, Lahti M, Braeken M, de Rooij SR, Entringer S, et al. Prenatal developmental origins of behavior and mental health: The influence of maternal stress in pregnancy. Neurosci Biobehav Rev. 2020 Oct;117:26-64.

2. Hermes M, Antonow-Schlorke I, Hollstein D, Kuehnel S, Rakers F, Frauendorf V, et al. Maternal psychosocial stress during early gestation impairs fetal structural brain development in sheep. Stress. 2020 Mar;23(2):233-42.

3. Moisiadis VG, Matthews SG. Glucocorticoids and fetal programming part 1: Outcomes. Nat Rev Endocrinol. $2014 \mathrm{Jul} ; 10(7): 391-402$.

4. Rakers F, Rupprecht S, Dreiling M, Bergmeier C, Witte OW, Schwab M. Transfer of maternal psychosocial stress to the fetus. Neurosci Biobehav Rev. 2017 Feb 22.

5. Moisiadis VG, Matthews SG. Glucocorticoids and fetal programming part 2: Mechanisms. Nat Rev Endocrinol. $2014 \mathrm{Jul} ; 10(7): 403-11$.

6. Mulkey SB, du Plessis AJ. Autonomic nervous system development and its impact on neuropsychiatric outcome. Pediatr Res. 2019 Jan;85(2):120-6.

7. Chrousos GP. Stress and disorders of the stress system. Nat Rev Endocrinol. 2009 Jul;5(7):374-81.

8. Glover V, O'Connor TG, O'Donnell K. Prenatal stress and the programming of the HPA axis. Neurosci Biobehav Rev. 2010 Sep;35(1):17-22.

9. Alexander N, Rosenlöcher F, Stalder T, Linke J, Distler W, Morgner J, et al. Impact of antenatal synthetic glucocorticoid exposure on endocrine stress reactivity in term-born children. The Journal of Clinical Endocrinology \& Metabolism. 2012;97(10):3538-44.

10. Ilg L, Kirschbaum C, Li SC, Rosenlocher F, Miller R, Alexander N. Persistent Effects of Antenatal Synthetic Glucocorticoids on Endocrine Stress Reactivity From Childhood to Adolescence. J Clin Endocrinol Metab. 2019 Mar 1;104(3):827-34.

11. Schleussner E, Möller A, Groß W, Kähler C, Möller U, Richter S, et al. Maternal and fetal side effects of tocolysis using transdermal nitroglycerin or intravenous fenoterol combined with magnesium sulfate. European Journal of Obstetrics \& Gynecology and Reproductive Biology. 2003;106(1):14-9.

12. Kromeyer-Hauschild K, Wabitsch M, Kunze D, Geller F, Geiß HC, Hesse V, et al. Perzentile für den Body-mass-Index für das Kindes- und Jugendalter unter Heranziehung verschiedener deutscher Stichproben. Monatsschrift Kinderheilkunde. 2001 2001/08/01;149(8):807-18.

13. Buske-Kirschbaum A, Jobst S, Wustmans A, Kirschbaum C, Rauh W, Hellhammer D. Attenuated free cortisol response to psychosocial stress in children with atopic dermatitis. Psychosom Med. 1997 JulAug;59(4):419-26.

14. Kirschbaum C, Pirke K-M, Hellhammer DH. The 'Trier Social Stress Test'-a tool for investigating psychobiological stress responses in a laboratory setting. Neuropsychobiology. 1993;28(1-2):76-81. 
15. Buske-Kirschbaum A, Krieger S, Wilkes C, Rauh W, Weiss S, Hellhammer DH. Hypothalamic-pituitaryadrenal axis function and the cellular immune response in former preterm children. J Clin Endocrinol Metab. 2007 Sep;92(9):3429-35.

16. Wu J, Phillip TM, Doretto V, van Noordt S, Chaplin TM, Hommer RE, et al. An inactive control of the 'Trier Social Stress Test' for Youth 10-17 years: Neuroendocrine, cardiac, and subjective responses. Psychoneuroendocrinology. 2019 Jun;104:152-64.

17. Kudielka BM, Buske-Kirschbaum A, Hellhammer DH, Kirschbaum C. HPA axis responses to laboratory psychosocial stress in healthy elderly adults, younger adults, and children: impact of age and gender. Psychoneuroendocrinology. 2004 2004/01/01/;29(1):83-98.

18. Nater UM, Rohleder N. Salivary alpha-amylase as a non-invasive biomarker for the sympathetic nervous system: current state of research. Psychoneuroendocrinology. 2009 May;34(4):486-96.

19. Malik M. Heart rate variability: Standards of measurement, physiological interpretation, and clinical use: Task force of the European Society of Cardiology and the North American Society for Pacing and Electrophysiology. Annals of Noninvasive Electrocardiology. 1996;1(2):151-81.

20. Frasch MG, Muller T, Wicher C, Weiss C, Lohle M, Schwab K, et al. Fetal body weight and the development of the control of the cardiovascular system in fetal sheep. J Physiol. 2007 Mar 15;579(Pt 3):893907.

21. Pagani M, Somers V, Furlan R, Dell'Orto S, Conway J, Baselli G, et al. Changes in autonomic regulation induced by physical training in mild hypertension. Hypertension. 1988;12(6):600-10.

22. Bulheller S, Häcker SO. Coloured Progressive Matrices (CPM). (Deutsche Bearbeitung und Normierung nach J. C. Raven.). : Pearson Assessment, Frankfurt

2002.

23. Döpfner M, Lehmkuhl G. Diagnostik-System für psychische Störungen im Kindes-und Jugendalter nach ICD-10 und DSM-IV. Bern: Huber Verlag. 1998.

24. Schwab M, Schmidt K, Roedel M, Mueller T, Schubert H, Anwar MA. Non-linear changes of electrocortical activity after antenatal betamethasone treatment in fetal sheep. The Journal of Physiology. 2001;531(2):535-43.

25. Cohen E, Wong FY, Wallace EM, Mockler JC, Odoi A, Hollis S, et al. EEG power spectrum maturation in preterm fetal growth restricted infants. Brain Res. 2018 2018/01//;1678:180-6.

26. Szeto HH, Vo TDH, Dwyer G, Dogramajian ME, Cox MJ, Senger G. The ontogeny of fetal lamb electrocortical activity: A power spectral analysis. American Journal of Obstetrics and Gynecology. 1985 1985/10/15/;153(4):462-6.

27. Hedges LV. Effect Sizes in Cluster-Randomized Designs. Journal of Educational and Behavioral Statistics. 2007 2007/12/01;32(4):341-70.

28. Grzeskowiak LE, Grivell RM, Mol BW. Trends in receipt of single and repeat courses of antenatal corticosteroid administration among preterm and term births: A retrospective cohort study. Australian and New Zealand Journal of Obstetrics and Gynaecology. 2017;57(6):643-50.

29. Schaffer L, Luzi F, Burkhardt T, Rauh M, Beinder E. Antenatal betamethasone administration alters stress physiology in healthy neonates. Obstet Gynecol. 2009 May;113(5):1082-8.

30. Sloboda DM, Moss TJ, Li S, Doherty D, Nitsos I, Challis JR, et al. Prenatal betamethasone exposure results in pituitary-adrenal hyporesponsiveness in adult sheep. Am J Physiol Endocrinol Metab. 2007 Jan;292(1):E61-70. 
31. Karemaker R, Kavelaars A, ter Wolbeek M, Tersteeg-Kamperman M, Baerts W, Veen S, et al. Neonatal Dexamethasone Treatment for Chronic Lung Disease of Prematurity Alters the Hypothalamus-PituitaryAdrenal Axis and Immune System Activity at School Age. Pediatrics. 2008;121(4):e870.

32. Erni K, Shaqiri-Emini L, La Marca R, Zimmermann R, Ehlert U. Psychobiological effects of prenatal glucocorticoid exposure in 10-year-old-children. Front Psychiatry. 2012;3:104.

33. Dalziel SR, Walker NK, Parag V, Mantell C, Rea HH, Rodgers A, et al. Cardiovascular risk factors after antenatal exposure to betamethasone: 30-year follow-up of a randomised controlled trial. Lancet. 2005 May 28-Jun 3;365(9474):1856-62.

34. Meuwese CL, Euser AM, Ballieux BE, van Vliet HA, Finken MJ, Walther FJ, et al. Growth-restricted preterm newborns are predisposed to functional adrenal hyperandrogenism in adult life. Eur J Endocrinol. 2010 Oct;163(4):681-9.

35. Camm AJ, Malik M, Bigger JT, Breithardt G, Cerutti S, Cohen R, et al. Heart rate variability: standards of measurement, physiological interpretation and clinical use. Task Force of the European Society of Cardiology and the North American Society of Pacing and Electrophysiology. 1996.

36. Xhyheri B, Manfrini O, Mazzolini M, Pizzi C, Bugiardini R. Heart rate variability today. Prog Cardiovasc Dis. 2012 Nov-Dec;55(3):321-31.

37. Billman GE. The LF/HF ratio does not accurately measure cardiac sympatho-vagal balance. Frontiers in physiology. 2013;4:26.

38. Dalziel SR, Lim VK, Lambert A, McCarthy D, Parag V, Rodgers A, et al. Antenatal exposure to betamethasone: psychological functioning and health related quality of life 31 years after inclusion in randomised controlled trial. BMJ. 2005 Sep 24;331(7518):665.

39. Dessens AB, Haas HS, Koppe JG. Twenty-year follow-up of antenatal corticosteroid treatment. Pediatrics. 2000 Jun;105(6):E77.

40. Doyle LW, Ford GW, Rickards AL, Kelly EA, Davis NM, Callanan C, et al. Antenatal corticosteroids and outcome at 14 years of age in children with birth weight less than 1501 grams. Pediatrics. 2000 Jul;106(1):E2.

41. MacArthur BA, Howie RN, Dezoete JA, Elkins J. Cognitive and Psychosocial Development of 4-Year-Old Children Whose Mothers Were Treated Antenatally with Betamethasone. Pediatrics. 1981;68(5):638-43.

42. Schmand B, Neuvel J, Smolders-de Haas H, Hoeks J, Treffers PE, Koppe JG. Psychological development of children who were treated antenatally with corticosteroids to prevent respiratory distress syndrome. Pediatrics. 1990 Jul;86(1):58-64.

43. Räikkönen K, Gissler M, Kajantie E. Associations Between Maternal Antenatal Corticosteroid Treatment and Mental and Behavioral Disorders in Children. JAMA. 2020;323(19):1924-33.

44. Bell AH, McClure B, McCullagh P, McClelland R. Spectral edge frequency of the EEG in healthy neonates and variation with behavioural state. Neonatology. 1991;60(2):69-74.

45. Cragg L, Kovacevic N, McIntosh AR, Poulsen C, Martinu K, Leonard G, et al. Maturation of EEG power spectra in early adolescence: a longitudinal study. Developmental Science. 2011;14(5):935-43.

46. Sherman D, Walterspacher D. Electroencephalography. Encyclopedia of Medical Devices and Instrumentation; 2006.

47. Shany E, Berger I, Goldberg O, Karplus M, Gilat S, Benzaquen O, et al. Do Prenatal Corticosteroids Affect Brain Maturation of the Premature Infant? An Electroencephalography Study. Clin EEG Neurosci. 2017 Mar;48(2):79-87. 
48. Schwab M, Schmidt K, Roedel M, Mueller T, Schubert H, Anwar MA, et al. Non-linear changes of electrocortical activity after antenatal betamethasone treatment in fetal sheep. J Physiol. 2001 Mar 1;531(Pt 2):535-43.

49. Davidson JO, Quaedackers JS, George SA, Gunn AJ, Bennet L. Maternal dexamethasone and EEG hyperactivity in preterm fetal sheep. J Physiol. 2011 Aug 1;589(Pt 15):3823-35.

50. Karemaker R, Karemaker JM, Kavelaars A, Tersteeg-Kamperman M, Baerts W, Veen S, et al. Effects of neonatal dexamethasone treatment on the cardiovascular stress response of children at school age. Pediatrics. 2008 Nov;122(5):978-87.

51. Luu TM, Rehman Mian MO, Nuyt AM. Long-Term Impact of Preterm Birth: Neurodevelopmental and Physical Health Outcomes. Clin Perinatol. 2017 Jun;44(2):305-14.

52. Russell AE, Ford T, Williams R, Russell G. The Association Between Socioeconomic Disadvantage and Attention Deficit/Hyperactivity Disorder (ADHD): A Systematic Review. Child Psychiatry Hum Dev. 2016 Jun;47(3):440-58.

53. Turkheimer E, Haley A, Waldron M, D'Onofrio B, Gottesman, II. Socioeconomic status modifies heritability of IQ in young children. Psychol Sci. 2003 Nov;14(6):623-8.

54. Piccolo LdR, Sbicigo JB, Grassi-Oliveira R, Fumagalli de Salles J. Do socioeconomic status and stress reactivity really impact neurocognitive performance? Psychology \& Neuroscience. 2014;7(4):567.

\section{Legends of Supporting Figures}

Figure S1: Flowchart of the study recruitment process

Figure S2: Stress response of the autonomic nervous system (ANS) expressed as the LF/HF ratio of the heart rate variability during the TSST-C (timepoints T1-T5) in children exposed to antenatal betamethasone (BM, solid line) and in controls (dashed line). Data expressed as mean with $95 \%$ confidence interval.

Figure S3: Cognitive performance (Intelligence Quotient, left) and ADHD core symptoms (Diagnostic System for Psychiatric Disorders in Children and Adolescents II [DISYPS II] score on stanine scale, right) in children exposed to antenatal betamethasone (BM, solid line) and in controls (dashed line). Data expressed as mean with $95 \%$ confidence interval.

Figure S4: Electrocortical activity expressed as the spectral edge frequency (SEF) of the EEG at the temporal and occipital electrodes during the TSST-C (timepoints T1-T5) in children exposed to antenatal betamethasone (BM, solid line) and in controls (dashed line). Data expressed as mean with $95 \%$ confidence interval.

\section{Hosted file}

Table 1 - Demographic Data.docx available at https://authorea.com/users/450989/articles/ 549245-impact-of-antenatal-glucocorticoid-exposure-on-the-activity-of-the-stress-systemcognition-and-behavior-in-8-to-9-year-old-children-a-clinical-cohort-study

\section{Hosted file}

Table 2 - Outcome Summary.docx available at https://authorea.com/users/450989/articles/ 549245-impact-of-antenatal-glucocorticoid-exposure-on-the-activity-of-the-stress-systemcognition-and-behavior-in-8-to-9-year-old-children-a-clinical-cohort-study 

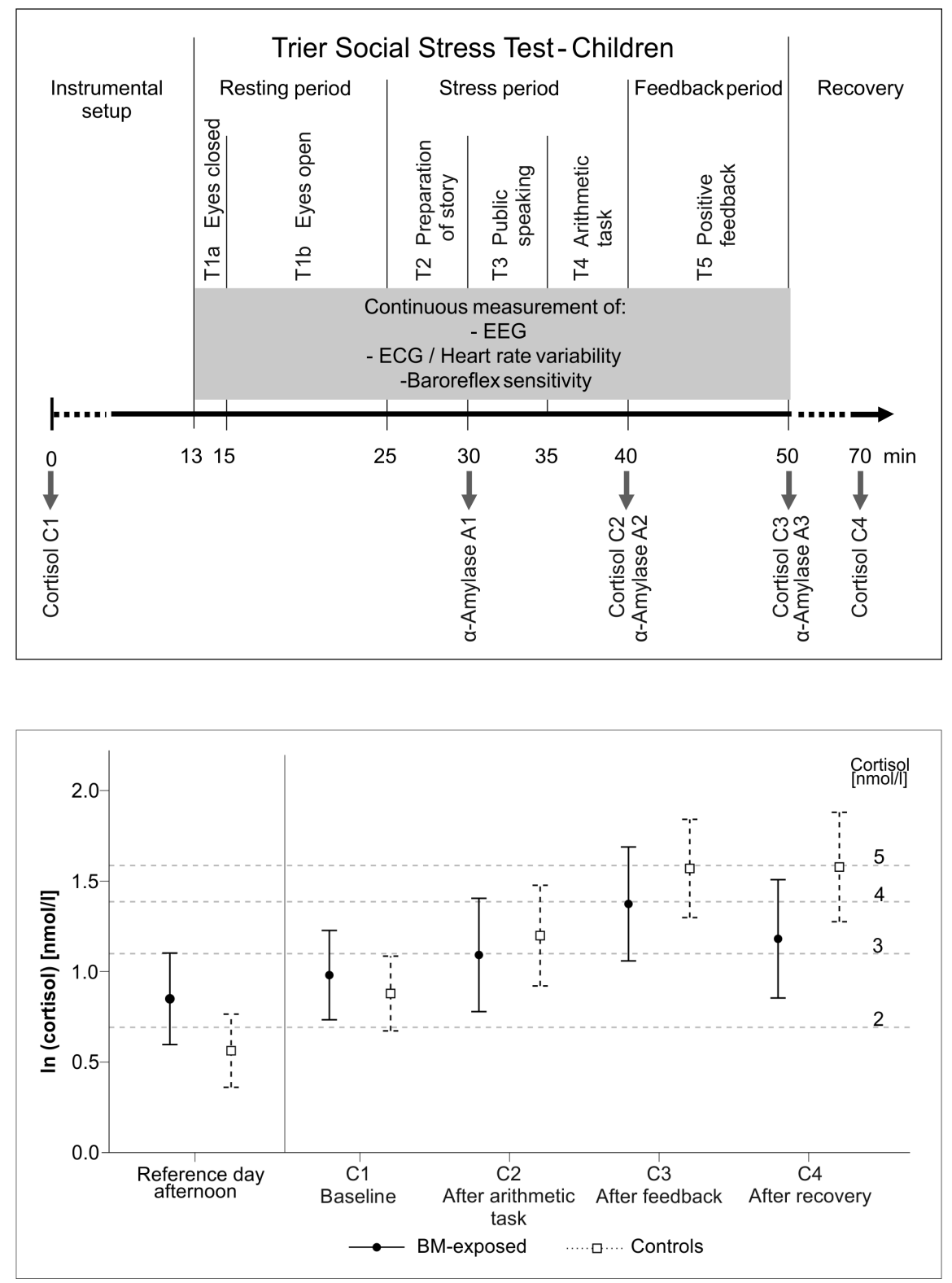